\title{
FGFR3 and TP53 mutations in urothelial cell carcinoma: two molecular pathways and diagnostic value
} Ashraf Bakkar

\author{
Address: Biochemistry Department, Sinai University, Cairo, Egypt \\ from 2009 American University in Cairo Research Conference \\ Cairo, Egypt. 5 April 2009 \\ Published: I July 2009 \\ BMC Proceedings 2009, 3(Suppl 3):07
}

This abstract is available from: http://www.biomedcentral.com/I753-656I/3/S3/O7

(C) 2009 Bakkar; licensee BioMed Central Ltd.

FGFR3 and TP53 mutations are frequent in superficial papillary and invasive disease urothelial cell carcinoma respectively. This high frequency of FGFR3 and TP53 mutations, led us to compare these two genetic alteration in 81 bladder cancer tumors of all stages and grades at initial diagnosis, to determine whether they correspond to different disease pathways and could be used for molecular classification of these tumors. Moreover, the role and the position of FGFR3 mutations in the current models of bladder carcinogenesis need to be clarified. We used denaturing high-performance liquid chromatography and sequencing to screen for FGFR3 and TP53 mutations in 81 newly diagnosed urothelial cell carcinomas. FGFR3 mutations were associated with low-stage $(P<0.0001)$, lowgrade $(P<0.008)$ tumors, whereas TP53 mutations were associated with high-stage $(P<0.003)$, high-grade $(P<$ $0.02)$ tumors. Mutations in these two genes were almost mutually exclusive. Our results suggest that FGFR3 and TP53 mutations define separate pathways at initial diagnosis of urothelial cell carcinoma. On the other hand, given the high frequency of FGFR3 mutations and the possible implication of this receptor in the development of UCC, it was important to develop a simple, fast, and reliable method to identify these mutations in greater detail as a potential tool for the diagnosis and follow-up of UCC patients. Ten different FGFR3 mutations have been described in UCC, but 4 of them (R248C, S249C, G372C, and $\mathrm{Y} 375 \mathrm{C}$ ) account for $95 \%$ of cases. Therefore in this study we developed an allele specific PCR (AS-PCR) for the detection of these mutations in tumors and urine. For the 95 tumors examined, AS-PCR had a specificity of $90 \%$ and a sensitivity of $88 \%$. The test correctly identifies FGFR3 mutations in the 20 tumors and the corresponding urine samples investigated. However, because our study was carried out on primary tumors rather on recurrent tumors, which are usually smaller, a prospective study of the diagnostic value of this test for the detection of recurrences should be undertaken. 